\title{
Orientational correlations in suspension of rodlike colloidal macroparticles
}

\author{
A. C. Brańka \\ Institute of Molecular Physics, Polish Academy of Sciences \\ Smoluchowskiego 17/19, 60-179 Poznań, Poland
}

\begin{abstract}
Orientational correlations of rodlike colloidal particles interacting via a two-site Yukawa potential are investigated using Brownian dynamics computer simulations. It is found that the orientational relaxation proceeds in three different regimes: very short-time regime, diffusive and long-time nondiffusive. An influence of particle concentration, the one-particle diffusion tensor, and particle elongation on these regimes is discussed.
\end{abstract}

\section{Introduction}

Colloidal suspensions are found in many industrial processes and consumer products. These are atomic or molecular liquids that have large solid particles of $c a$. $0.1 \mu m$ diameter suspended in them. These occur in, for exampie, foods, paints, inks, and many other frequently encountered products. There is considerably industrial and academic interest in understanding the relationship between the microscopic behaviour of these suspensions and their macroscopic physical properties which will enable a more systematic improvement of their properties and usefulness to be made.

The mass of the colloidal particle is much greater than that of the solvent particle and consequently, in colloidal fluids one is faced with a large spread of important time scales that are well separated [1] [2], Thus, on the macroparticle time scale (which is practically the only relevant time scale for colloidal systems) the solvent can be approximated well as a continuum fluid medium. The macroparticles interact through both interparticle colloidal forces and hydrodynamic forces mediated via the continuum fluid. The macroparticles also receive fluctuating Brownian forces which arise from the apparently random thermal motion of the fluid. The interplay between these different interactions is particularly complex in colloids composed of anisotropic particles. The microscopic modelling of such colloids is considerably less developed than colloidal suspensions composed of spherical macroparticles. Most of the numerical and theoretical studies to date have been concentrated on structural properties and/or properties of highly anisotropic rodlike particles, e.g., the tabacco-mosaic virus (TMV). There are, however many inorganic and polymeric colloidal suspensions 
that consist of compact, moderately nonspherical axisymmetric molecules [3], [4] [5]. For such particles, there is still little known about their dynamical behaviour at a molecular level.

A fundamental aspect underpinning all colloidal behaviour is diffusion of colloidal particles. In this report we focus on rotational diffusivity of moderately nonspherical interacting colloidal particles. The rotational diffusity, unlike translational one is less amenable to experimental studies. Also a purely microscopic theory of dynamical correlations, which determine particle diffusivity, is just beginning and most of analytical treatments is able to deal only with fairly simplify models. A powerful way to get direct insight into dynamical correlations of colloidal fluid are Brownian dynamics (BD) computer simulations [6] [7].

In this report we present the BD simulations for a molecular colloidal fluid of nonspherical, axisymmetric molecules interacting via a two-site Yukawa potential. The Yukawa potential, being the electrostatic part of the DVLO potential, is considered to be a realistic model for charged stabilized colloidal suspensions [8] [9] [10]. In our previous studies [11] we found that the relaxation of the orientational correlation has three qualitatively different regimes: free particle diffusion at very short-time, diffusive for intermediate times, and a nondiffusive long-time relaxation. Recently, Kirchhoff, Löwen and Klein [12] have found similar behaviour in fluid of slender particles interacting by a Yukawa segment-segment potential (the model particularly designed for aqueous suspensions of the TMV). The existence of three different relaxation regimes of the orientational correlation of nonspherical colloidal particles questions a commonly shearing view that the relaxation is purely diffusive [13] [14].

We have performed further calculations on the two-site Yukawa colloidal fluid in order to get better characterization of the orientational relaxation regimes. In particular we seek for an influence of the particle elongation on the range of the diffusive regimes.

\section{Brownian dynamics simulations}

We consider a system of $N$ rigid linear molecules suspended in an incompressible fluid of viscosity $\eta_{0}$. The orientation of the molecules is defined by a unit vector, e, along the molecular axis. The molecule is defined by two sites separated by the distance, L, and the separation distance is a parameter defining asymmetry of the molecule. The site-site interaction between different molecules $i$ and $j$, is assumed to be pairwise and the total potential energy in a given configuration is,

$$
V\left(\mathbf{r}_{i j}, \mathbf{e}_{i}, \mathbf{e}_{j}\right)=\sum_{a} \sum_{b} u\left(r_{a b}\right),
$$


where $r_{a b}=\left\|\mathbf{r}_{i a}-\mathbf{r}_{j b}\right\|$ is the site-site distance. The site-site potential is modeled by a Yukawa potential,

$$
u(r)=U_{0} \exp [-\lambda(r-1)] / r,
$$

where $U_{0}$ sets the energy scale and $\lambda$ is the screening parameter characterizing the steepness and range of the potential. The many-body hydrodynamic interactions are neglected in our model and the interaction of the molecule with the solvent is represented by a one-particle diffusion tensor. For rodlike particle, the diffusion tensor contains four nonzero components: two translational, $D_{\perp}^{t}, D_{\|}^{t}$, and two rotational $D_{\perp}^{r}, D_{\|}^{r}$, coefficients. (The subscripts $\|$ and $\perp$ denote parallel and perpendicular to the molecular axis, respectively.) The rotational diffusion around the symmetry axis, $D_{\|}^{r}$, can be safely ignored and the $D_{\perp}^{r}$ coefficient will subsequently be denoted as $D_{0}^{R}$.

The one-particle diffusion tensor is available only for few molecular models, e.g. ellipsoids with the stick hydrodynamic boundary conditions [15] [3]. In general, values of the diffusion coefficients depend markedly from the size and shape of the molecule, solvent properties [16] [17], and the type of hydrodynamic boundary conditions used for their calculations [18] [19] [20]. To get some information on this dependence we shall use two different values of $D_{\|}^{t}=1$ and 5 .

In order to study a system of particles which can rotate in time the diffusion tensor has to be transformed from the molecular-fixed frame to the laboratory-fixed frame. This transformation leads to the following form for the translational diffusion tensor $\mathbf{D}^{T}$ in the laboratory-fixed frame:

$$
D_{\alpha \beta}^{T}=D_{\perp}^{t} \delta_{\alpha \beta}+e_{\alpha} e_{\beta}\left(D_{\|}^{t}-D_{\perp}^{t}\right)
$$

where $\mathrm{e}_{\alpha}$ is the $\alpha$ component of the unit vector e and $\delta_{\alpha \beta}$ is the Kronecker delta. As molecule rotates, the tensor $\mathbf{D}^{T}$ changes in time. This means that the translational motion of a rod-like particle depends on its orientation what is a result of different diffusivities in the directions parallel and normal to the rod axis. The expression (3) can be rearranged into the form in which a part of the tensor is independent of molecular orientation:

$$
D_{\alpha \beta}^{T}=D_{I} \delta_{\alpha \beta}+D_{A}\left(e_{\alpha} e_{\beta}-1 / 3 \delta_{\alpha \beta}\right),
$$

where $D_{A} \equiv\left(D_{\|}^{t}-D_{\perp}^{t}\right)$ and $D_{I} \equiv\left(D_{\|}^{t}+2 D_{\perp}^{t}\right) / 3$ is the isotropic translational diffusion coefficient $\left(1 / 3\right.$ of the trace of the $\left.\mathbf{D}^{T}\right)$.

For the simulation of the Brownian motion of molecules we adopt an algorithm proposed by Dickinson [21] [22] which for our system can be 
written as follows:

$$
\begin{gathered}
\Delta r_{i \alpha}=\frac{\Delta t}{T} \sum_{\beta} D_{i \alpha \beta}^{T} F_{i \beta}+\delta r_{i \alpha}, \\
\Delta \varphi_{i \alpha}=\frac{\Delta t}{T} D_{0}^{R} \tau_{i \alpha}+\delta \varphi_{i \alpha},
\end{gathered}
$$

where $i=1, \ldots, \mathrm{N}$ labels the particles and $\alpha, \beta$ indicates the cartesian coordinates $(x, y, z$ or $1,2,3) . F_{i \alpha}, \tau_{i, \alpha}$ are the net force and torque acting in direction ct on the particle, i. $T$ stands for the temperature. $\delta \varphi_{i \alpha}$ is a random orientational displacement which is sampled from a Gaussion distribution of zero mean and variance

$$
<\delta \varphi_{\alpha}^{2}>=2 D_{0}^{R} \Delta t
$$

Similarly, $\delta r_{i \alpha}$ denotes a random displacement of the molecular center of mass. In this case, however the cartesian components of the displacement vector $\delta \mathbf{r}$, for a given molecule, are correlated in accordance with the $\mathbf{D}^{T}$ tensor and has to be sampled from a multivariate Gaussian distribution with zero mean and the variance-covariance matrix,

$$
<\delta r_{i \alpha} \delta r_{i \beta}>=2 D_{i \alpha \beta}^{T} \Delta t .
$$

All quantities are normalized to dimensionless forms, by choosing $\sigma, \sigma^{2} / D_{0}$ and $U_{0} / \sigma$ as the characteristic values for length, time and forces. Temperature is in $k_{B} / U_{0}$ and all the diffusion coefficients are in the $D_{0}$ units. The BD calculations were performed for a system of moderately nonspherical particles with $L=1.0$. The system consisted of $N=125$ molecules in a periodic boundary conditions and the basic simulation cell was a cube of volume $V$. Particle trajectories were generated according to the algorithm (5-8). During the simulations the center of mass of the system was fixed.

The screening parameter in the interaction potential was $\lambda=8$ and the reduced temperature was $T=1$, i.e., the values chose for the spherical particles in ref. [23] and used in ref. [11] for the case $L=0.5$. As the potential is strongly repulsive a sufficiently small time step has to be used. The actual value of $\Delta t$ in the simulations was $5 \times 10^{-5}$. The calculations were performed for a range of particle densities $\varrho=N / V$. The simulations started at low density and after long equilibration run of $10^{5}$ time steps the run of $10^{6} \Delta t$ (i.e., a period of $50 \sigma^{2} / D_{0}$ ) was made. Next, the system was gently driven to higher density where was equilibrated for $0.5 \times 10^{5} \Delta t$ and the basic run of $10^{6} \Delta t$ was performed from which the averages were calculated. A long, well equilibrated, runs are necessary to get, particularly at low densities, sufficient statistic to determine the dynamical properties. 


\section{Orientational correlation functions}

The orientational dynamics of an ensemble of noninteracting molecules is described by the Debye rotational diffusion equation [24]. The solution of this diffusion equation is in terms of the Legendre polynomials, $P_{l}(x)$, and the orientational ordering is characterized by the set of the exponentially decaying correlation functions,

$$
<P_{l}(\mathrm{e}(t) \mathrm{e}(0))>=\exp \left(-l(l+1) D_{0}^{R} t\right),
$$

with the single diffusion coefficient $D_{0}^{R}$.

In an ensemble of interacting Brownian particles one expects that the hydrodynamic and interparticle interactions will modify this pure exponential decay. In this case it is reasonable to defined the rotational correlation functions as

$$
C_{l}(t)=<P_{l}(\mathbf{e}(t) \mathbf{e}(0))>=\exp \left(-l(l+1) D_{l}^{R}(t) t\right),
$$

with a set of corresponding time-dependent diffusion 'coefficients', $D_{l}^{R}(t)$. The orientational motion is diffusive if $D_{l}^{R}(t)$ become independent of $t$ and I.

We have studied the first two orientational correlation functions by considering the two relations that follow from eq.(10),

$$
\begin{aligned}
& p_{1}=-\frac{1}{2} \ln \left\langle C_{1}>/ D_{0}^{R} .\right. \\
& p_{2}=-\frac{1}{6} \ln \left\langle C_{2}>/ D_{0}^{R} .\right.
\end{aligned}
$$

The typical time behaviour of these functions is plotted in Fig. 1. Figure, clearly shows that initially, both functions, increase linearly with time and are practically indistinguishable. The slope of the functions in the linear period is less than unit, what means, the orientational correlation functions can be well described, at least in this period, in terms of a modified rotational diffusion coefficient, $D_{r}^{R}$.

At longer times, however the slope for $1=1,2$ appears to saturate. The different behaviour of the orientation functions for different 1 demonstrates that the orientational relaxation is not diffusive for longer times.

Fig.2 shows also that for an initial, very short but observable time interval $(t<0.01)$, an decrease of the slope occurs from 1 to $D_{r}^{R} / D_{0}^{R}$. This means that initially the particles rotate as in a dilute solution, i.e., there exists an initial amount of unhindered rotation.

The coefficients $D_{r}^{R}$ calculated from the slope of the $\mathrm{p}_{1}$ over the time interval $0.01-0.3$, are shown in Fig.3 together with the results for $L=0.5$. Clearly, density dependence for suspension composed of longer molecules is stronger. Also in dilute region the density dependence of $D_{r}^{R}$ for more elongated particles can not be considered as a linear one. 


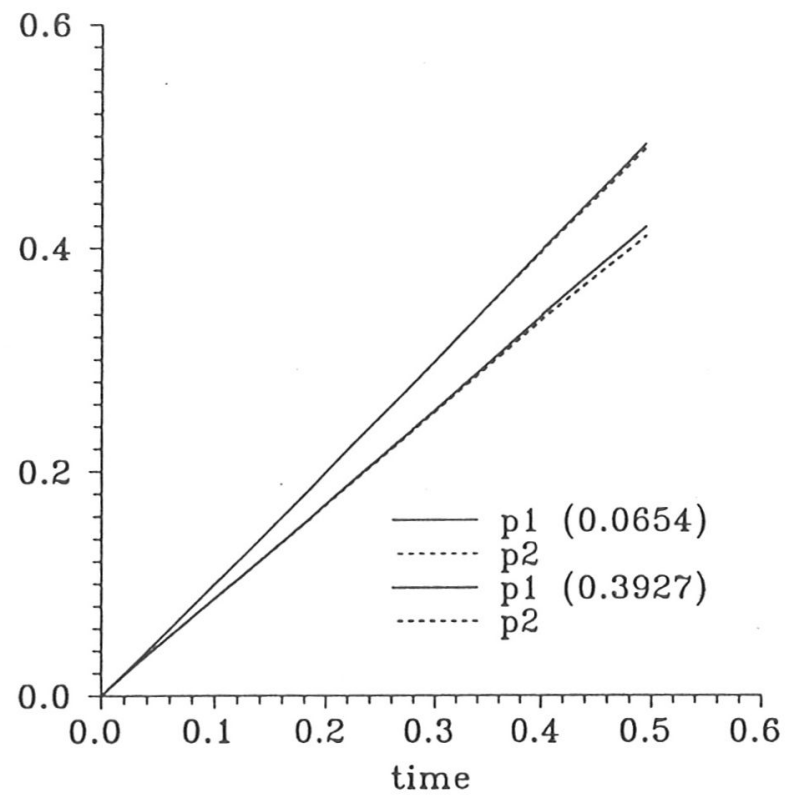

Fig.1 The time evolution of the rotational correlation functions (eqs. 12,13 ) at two concentrations. Results for suspension of the two-site Yukawa rod-like molecules with $L=1$.

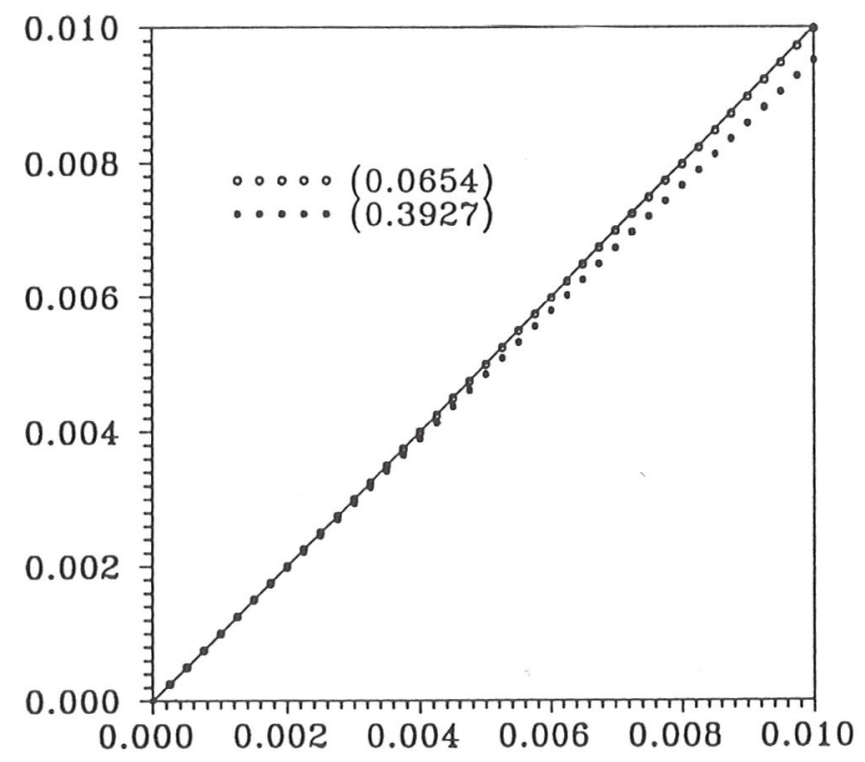

time

Fig.2 The rotational correlation functions (see Fig.1) at short times. The solid line is $D_{0}^{R} t$. 


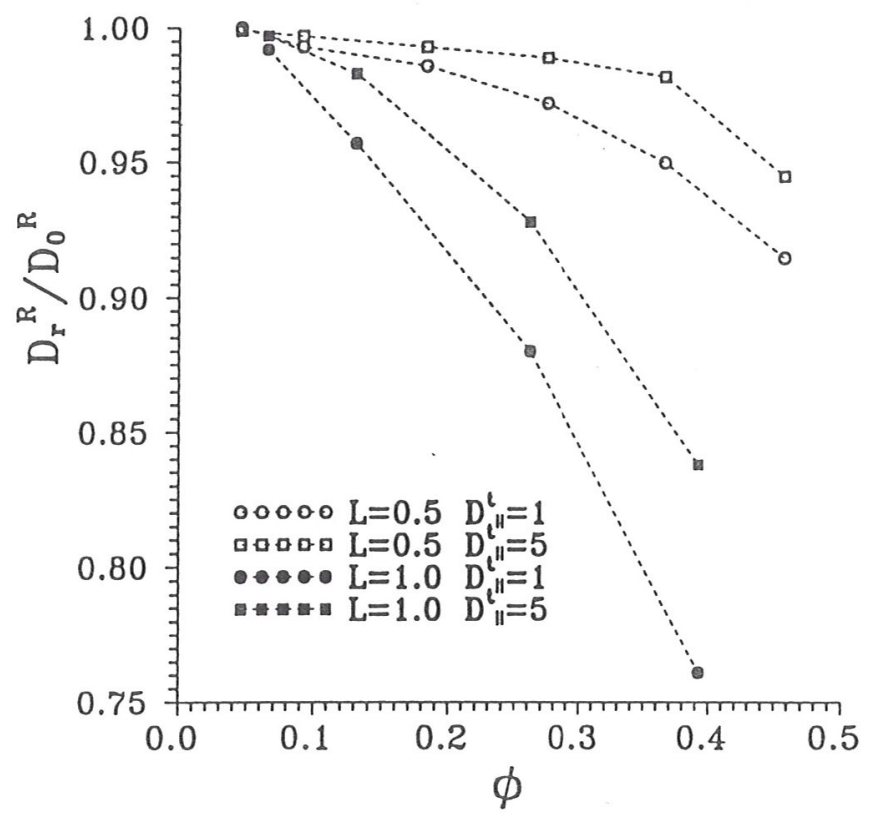

Fig.3 The rotational diffusivity vs concentration for different molecular anisotropies and one-particle diffusion coefficients. $\phi=v \varrho$ is a packing fraction and $v=\frac{\pi}{6}(1+3 L)$ is volume of a spheroid approximating a size of the Yukawa molecule.

\section{Conclusions}

In the work we have concentrated on rotational relaxation in colloidal suspension composed of moderately anisotropic particles. We have studied a two-site Yukawa molecular system with the site-site separation $L=1$ obeying Brownian dynamics. The model can be considered as a first approximation for charge rod-like particle suspension.

Our results confirm our previous observations for more compact (less anisotropic) molecules that rotational relaxation take place in three time stages: very short-time regime $t<t_{s}$, the linear or diffusive regime $t_{s}<$ $t<t_{m}$ characterized by an effective diffusion coefficient $D_{r}^{R}$, and long-time nondiffusive regime. The value of $t_{s}$ is less than 0.01 for all concentrations and we do not found any considerable influence of molecular anisotropy on this regime. The second regime and $t_{m}$ displays more density, $\mathrm{D}^{T}$, and $\mathrm{L}$ dependence. The density and $\mathrm{D}^{T}$ dependence of $D_{r}^{R}$ is stronger for longer molecules.

A weak concentration dependence of the rotational diffusion coefficient has been predicted by Jones [25] and recently demonstrated experimentally in a suspension of spherical particles possessing an intrinsic optical anisotropy [26]. Our calculations suggest that the effect should be stronger and better 
observable in a suspension of elongated particles. More experiments for carefully prepared and well-characterized suspensions of rodlike particles are needed to get a more comprehensive picture of the orientational relaxation regimes.

Acknowledgements. The work has been supported by the Polish Committee for Scientific Research (KBN) grant No. 8T11F 01008 p04. The calculations has been performed at Poznari Computer and Networking Centre.

\section{References}

[1] W. Hess and R. Klein, Adv. in Phys. 32, 173 (1983).

[2] W.B. Russel, D.A. Saville, and W.R. Schowalter, Colloidal Dispersions (Cambridge University Press, 1989).

[3] T.G.M. Van de Ven, Colloidal Hydrodynamics (Academic Press, 1988)

[4] M. Ocana, M. Anders, M. Martine, C.J. Serna, and E. Matijevic, J. Colloid Interface Sci. 16, 262 (1994).

[5] D.M.E. Thies-Weesie, A.P. Philipse, and S.J.M. Kluijtmans, J. Colloid Interface Sci. 174, 211 (1995).

[6] D.L. Ermak and J.A. McCamon, J. Chem. Phys. 69, 1352 (1978).

[7] M.P. Allen and D.J. Tildesley, Computer Simulation of Liquids (Clarendon Press, Oxford, 1987).

[8] J. Schneider, W. Hess and R. Klein, J.Phys. A 18, 1221 (1985).

[9] J. Schneider, D. Karrer, J.K.G. Dhont, and R. Klein, J. Chem. Phys.87, 3008 (1987).

[10] H. Löwen, Phys. Rev. Lett. 72, 424 (1994); J. Chem. Phys.100, 6738 (1994).

[11] A.C. Brańka and D.M. Heyes, Phys. Rev. E 50, 4810 (1994).

[12] Th. Kirchhoff, H. Löwen and R. Klein, Phys. Rev. E 53, 5011 (1996).

[13] M. Doi, I. Yanamoto, and F. Kano, J. Phys. Soc. Jap. 53, 3000 (1984).

[14] I. Bitsanis, H.T. Davies, and M. Tirrell, Macromolecules 21, 2824 (1988).

[15] F. Perrin, J. Phys. Radium 5, 33 (1934); 71 (1936). 
[16] T.P. Lodge, J. Phys. Chem. 97, 1480 (1993).

[17] F.W. Wiegel and P.F. Mijnlieff, Physica A 89, 385 (1977).

[18] C. Hu and R. Zwanzig, J. Chem. Phys. 60, 4354 (1974).

[19] D.R. Bauer, J.I. Brauman, and R. Pecora, J. Amer. Chem. Socity 96, 6840 (1974).

[20] R. B. Jones and R. Schmitz, Physica A 149, 373 (1988).

[21] E. Dickinson, J. Chem. Soc., Faraday Trans. 2, 81591 (1985).

[22] E. Dickinson, Chem. Soc. Rev. 14, 421 (1985).

[23] H. Löwen and G. Szamel, J. Phys. Condens. Matter. 5, 2295 (1993).

[24] P. Debye, Polar Molecules (Dover, New York, 1929).

[25] R. B. Jones, Physica A 150, 339 (1988).

[26] R. Piazza, V. Degiorgio, M. Corti, and J. Stavans, Phys. Rev.B 42, 4885 (1990). 